\title{
La conductancia estomática y su relación con la temperatura foliar y humedad del suelo en el cultivo del olivo (Olea europaea L.), en periodo de maduración de frutos, en zonas áridas. La Yarada, Tacna, Perú
}

\author{
Stomatal conductance and its relationship with leaf temperature \\ and soil moisture in olive cultivation (Olea europaea L.), in the period \\ of fruit ripening, in arid zones. La Yarada, Tacna, Perú
}

Edwin Pino V. ${ }^{*}$, Isaac Montalván D. ${ }^{1}$, Alissa Vera M. ${ }^{1}$, Lia Ramos F. ${ }^{2}$

\section{RESUMEN}

Este trabajo busca la relación entre la conductancia estomática, la temperatura foliar y la humedad del suelo en el cultivo del olivo (Olea europaea L.), en periodo de maduración de frutos, en la irrigación La Yarada, Tacna, Perú. Se trabajó en una parcela con olivos de 22 años de edad, variedad sevillana, en plena producción, con marco de plantación de 7 x 7 m. Se realizaron las mediciones en una primera etapa, en un árbol en forma puntual. En una segunda etapa, se utilizó un total de 12 árboles para medir la conductancia en forma intensiva. La temperatura y la conductancia estomática arrojaron un ajuste significativo, y latendencia es a mayor temperatura foliar mayor conductancia estomática. Asimismo, no existe correlación entre la conductancia estomática y el contenido de humedad volumétrico del suelo. Según medidas intensivas de conductancia estomática, se observa una tendencia típica de picos hacia las horas medias del día, y se encuentra un valor máximo de $411 \mathrm{mmoles} / \mathrm{m}^{2} \mathrm{~s}$, en este período de maduración de frutos en el olivo.

Palabras clave: conductancia estomática, temperatura foliar, humedad del suelo, olivo.

\begin{abstract}
This work seeks the relationship between stomatal conductance, leaf temperature and soil moisture in the cultivation of the olive tree (Olea europaea L.), in the period of fruit ripening, in La Yarada irrigation, Tacna, Peru. A plot with 22-year-old olive trees, variety from Seville, in full production, with a planting frame of $7 \times 7 \mathrm{~m}$ was used for the study. Measurements were made in the first stage, in a tree in a particular style. In the second stage, a total of 12 trees were used to measure conductance intensively. Temperature and stomatal conductance showed a significant adjustment, with a tendency to higher leaf temperature, greater stomatal conductance. Likewise, there is no correlation between the stomatal conductance and the volumetric moisture content of the soil. According to intensive measures of stomatal conductance, a typical tendency of peaks towards the average hours of the day is observed, finding a maximum value of 411 mmoles $/ \mathrm{m}^{2} \mathrm{~s}$, in this period of fruit ripening in the olive tree.
\end{abstract}

Keywords: stomatal conductance, leaf temperature, soil moisture, olive.

\section{Introducción}

Uno de los procesos de estrés hídrico abiótico de mayor importancia que limitan la productividad de los cultivos en todo el mundo es la sequía ( $\mathrm{Li}$ et al., 2015). Es de esperar que estas sequías aumenten en intensidad y frecuencia en varias regiones del mundo, como consecuencia del cambio climático (Lauer et al., 2012; Trenberth et al., 2014). Es notorio que en muchos lugares la escasez de agua es una limitante altamente significativa del desarrollo de los cultivos (Dell'Amico-Rodríguez y Morales-Guevara, 2017). La mortalidad inducida por la sequía en plantas leñosas es cada vez más alta (Mencuccini, 2014). Al ser organismos sésiles, las plantas están constantemente expuestas a diversos

\footnotetext{
1 Universidad Nacional Jorge Basadre Grohmann. Tacna, Perú.

2 Universidad Nacional Agraria La Molina. Lima, Perú.

* Autor por correspondencia: epino68@hotmail.com; epinov@unjbg.edu.pe
} 
tipos de estímulos ambientales. Para sobrevivir en condiciones ambientales desfavorables, han desarrollado estrategias con el fin de permitir un equilibrio entre el crecimiento, la reproducción y la supervivencia (Prasch y Sonnewald, 2015).

La mayoría de los cultivos, incluyendo el olivo (Olea europaea L.), son sensibles al estrés hídrico en sus diferentes fases de desarrollo (Nielsen y Nelson, 1998). El agua es uno de los factores más importantes en el desarrollo de los cultivos, y ello implica que la programación del riego es decisiva para incrementar el rendimiento y la calidad de la cosecha (Wang et al., 2011). Siendo el crecimiento de las plantas controlado por la fotosíntesis como un fenómeno clave entre los demás procesos, el estudio del estado interno y flujo del agua en ellas es muy importante (Ashraf y Harris, 2013).

La disponibilidad de agua en los suelos restringe la cantidad de carbono que se puede absorber y asimilar a la nueva biomasa. Es posible maximizar la fotosíntesis a una disponibilidad de agua dada, modificando la conductancia estomática (Manzoni et al., 2013).

En el pasado, las respuestas estomáticas generalmente se consideraban en relación con variables ambientales individuales, en parte porque las interacciones entre factores parecían difíciles de cuantificar de una manera simple. Se ha informado de una correlación lineal entre la conductancia estomática (g) y la tasa de asimilación de $\mathrm{CO}_{2}$, cuando se varió la fluencia de los fotones y cuando la capacidad fotosintética de las hojas se vio alterada por las condiciones de crecimiento, siempre que el $\mathrm{CO}_{2}$, la humedad del aire y la temperatura de las hojas fueron constantes (Ball et al., 1987). Sin embargo, la temperatura y la humedad no son consistentes en la naturaleza. La interrupción de la relación positiva entre la conductancia estomática y el potencial hídrico de las hojas a humedad constante del suelo indica claramente que el estado del agua de la raíz o del suelo afecta directamente la conductancia estomática, minimizando la posibilidad de un mecanismo de retroalimentación a través del estado del agua de la hoja en el cultivo de olivos (Giorio et al., 1999). El intercambio de gases en la hoja de olivos maduros (Olea europaea L.) se evidenció en una amplia gama de déficits de agua en el campo. La fotosíntesis de la hoja y la conductancia estomática respondieron diurna y estacionalmente a las variaciones en el estado hídrico de los árboles y la demanda de evaporación (Moriana et al., 2002). La transpiración de las copas de alto acoplamiento, como los olivares, se puede calcular diariamente con suficiente precisión mediante la ecuación de 'hoja grande' de Penman-Monteith, con un modelo de volumen diario (Orgaz et al., 2007).

La conductancia estomática históricamente se ha predicho casi exclusivamente utilizando modelos empíricos o fenomenológicos, como las familias de modelos Jarvis y Ball-Berry (Beerling, 2015; Ball et al., 1987). Tales modelos son perfectamente adecuados para la predicción a futuro en situaciones donde sus parámetros pueden estimarse con confianza. De hecho, continúan informando de las proyecciones de intercambio de gases en toda la comunidad de modelos. Los avances recientes han mejorado nuestra capacidad para modelar la conductancia estomática utilizando modelos basados en el proceso u optimalidad, y la investigación debe centrarse en cómo los estomas detectan la turgencia de la hoja y en cómo cuantificar los niveles de carbono del bajo potencial hídrico en la hoja (Buckley, 2017).

Las adaptaciones morfológicas y estructurales a nivel de la hoja para reducir la pérdida de agua se examinaron en cinco olivos (Olea europaea L.) cultivares de árboles (Arbequina, Blanqueta, Cobrançosa, Manzanilla y Negrinha) que crecen en condiciones de campo con poca disponibilidad de agua (Bacelar et al., 2004). Se encontraron diferencias genotípicas considerables entre los cultivares. Negrinha, Manzanilla y Cobrançosa tuvieron más adaptaciones foliares morfológicas y estructurales para proteger contra la pérdida de agua que las otras variedades.

La pérdida de conductancia hidráulica es un mecanismo importante para aumentar el control estomático de la transpiración bajo un secado progresivo del suelo. Otra visión implica que la creciente diferencia entre el potencial hídrico del suelo y las hojas durante el verano induce el cierre estomático y minimiza el riesgo de colapso del sistema conductor, disminuyendo la transpiración y reduciendo la conductancia hidráulica (Tognetti et al., 2009). Las respuestas estomáticas a la turgencia foliar integran los efectos de varios otros factores. Muchas respuestas estomáticas surgen del efecto del potencial hídrico de la hoja, que integra las influencias de numerosas variables ambientales y vegetales (Buckley, 2017). 
En tal sentido, este trabajo tiene como objetivo buscar la relación entre la conductancia estomática, la temperatura foliar y la humedad del suelo en el cultivo del olivo (Olea europaea L.), en periodo de maduración de frutos, en la irrigación La Yarada, Tacna, Perú, ubicada en la cabecera del desierto de Atacama, caracterizado por su extrema aridez.

\section{Metodología}

Este trabajo fue realizado en condiciones de campo en la irrigación La Yarada, Tacna, Perú. En la Figura 1 se muestra la ubicación de la zona de estudio. Se trabajó en una parcela con olivos de 22 años de edad, variedad sevillana, en plena producción, instalados en un marco de plantación de 7 x $7 \mathrm{~m}$. El olivo en esa región es de la familia Oleaceae, origen Mediterráneo (costa de Siria, Líbano e Israel), variedades Sevillana, Ascolana y Liguria. En cuanto al período vegetativo, a los 3 años del injerto se obtiene la primera cosecha. En la Figura 2a se muestra la plantación donde se realizaron las mediciones en una primera etapa, en una planta específica. En la Figura 2 b se observa la plantación donde se hicieron las mediciones en una segunda etapa. Se utilizó un total de 12 árboles para medir la conductancia entre las 11:00 y 14:00 horas en forma intensiva.

El agua utilizada en el riego tiene una CE de $1,35 \mathrm{mmhos} / \mathrm{cm}$ con un $\mathrm{pH}$ en 7,13. Ambos se encuentran en el rango permisible para el cultivo del olivo. El suelo es franco arenoso, en los cuatro estratos estudiados, de $20 \mathrm{~cm}$ de espesor cada uno, con un perfil de $80 \mathrm{~cm}$ de profundidad. Tiene una densidad aparente promedio de $1,65 \mathrm{gr} / \mathrm{cm}^{3}$, la cual se obtuvo en campo con 5 repeticiones en el estrato de 0 a $20 \mathrm{~cm}$ de profundidad, considerando esta la capa donde se encuentra la mayor concentración radicular. Se realizó una prueba de infiltración usando cilindros infiltrómetros, y se lograron tasas de infiltración muy altas en el inicio de la prueba, como se puede observar en la Figura 3. El modelo de ajuste obtenido es: $\mathrm{i}=329,74 \mathrm{~T}^{-0,378}$ (i en $\mathrm{mm} / \mathrm{hr}$, $\mathrm{T}$ en minutos), con un coeficiente de correlación $\mathrm{r}^{2}$ de 0,82 , coeficientes típicos de suelos arenosos a franco arenosos.

Eln clima en la zona es húmedo en invierno, cuando abundan las neblinas o camanchacas, y semicálido el resto del año. Sus temperaturas promedio mensuales son de $24,6{ }^{\circ} \mathrm{C}$ en febrero como máxima y la mínima $13,6^{\circ} \mathrm{C}$ en julio. Las precipitaciones son prácticamente nulas, típico comportamiento de regiones desérticas (Pino et al., 2017). Estas características hacen que en la zona se presente un clima templado, desértico y con amplitud térmica moderada a lo largo de los años. La precipitación media acumulada anual está en el orden de los 33,4 mm. Por eso es considerada como una región con pocas lluvias y de clima seco (esta zona forma parte del desierto de Atacama).
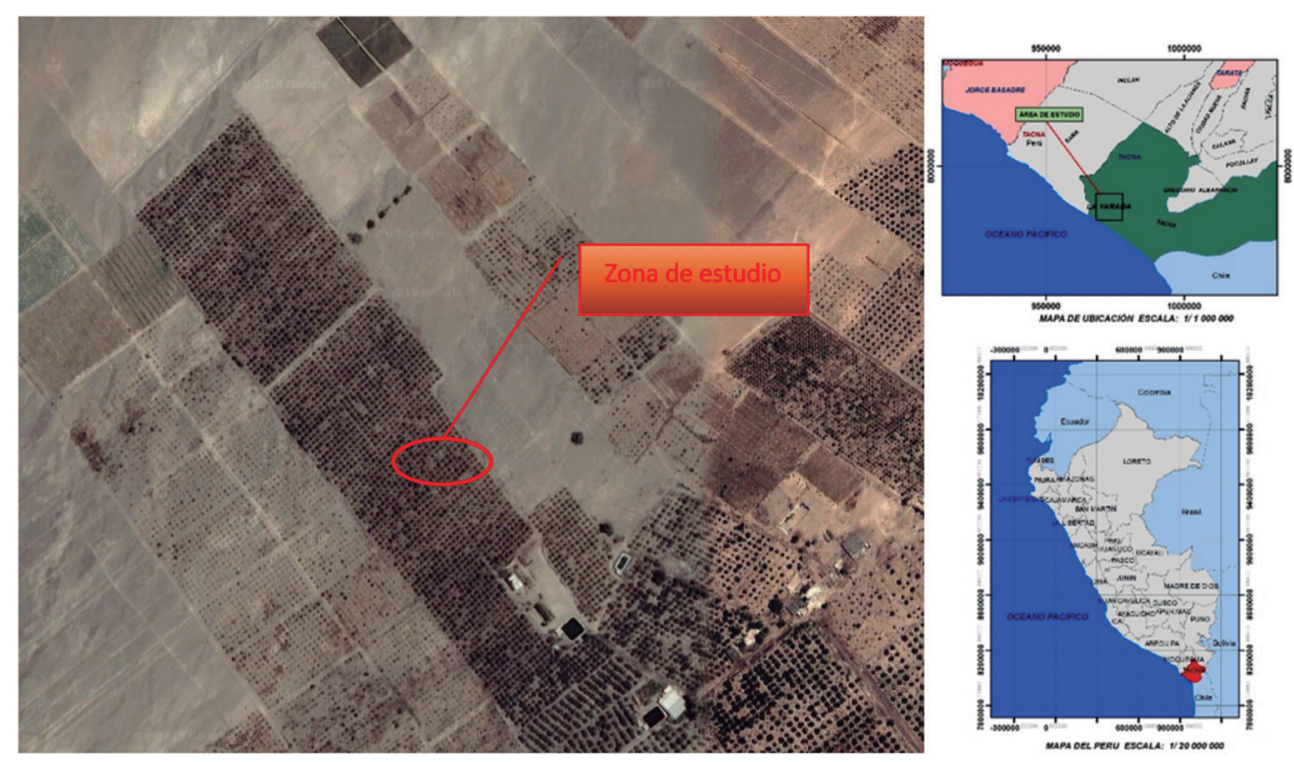

Figura 1. Ubicación de la zona de estudio. 


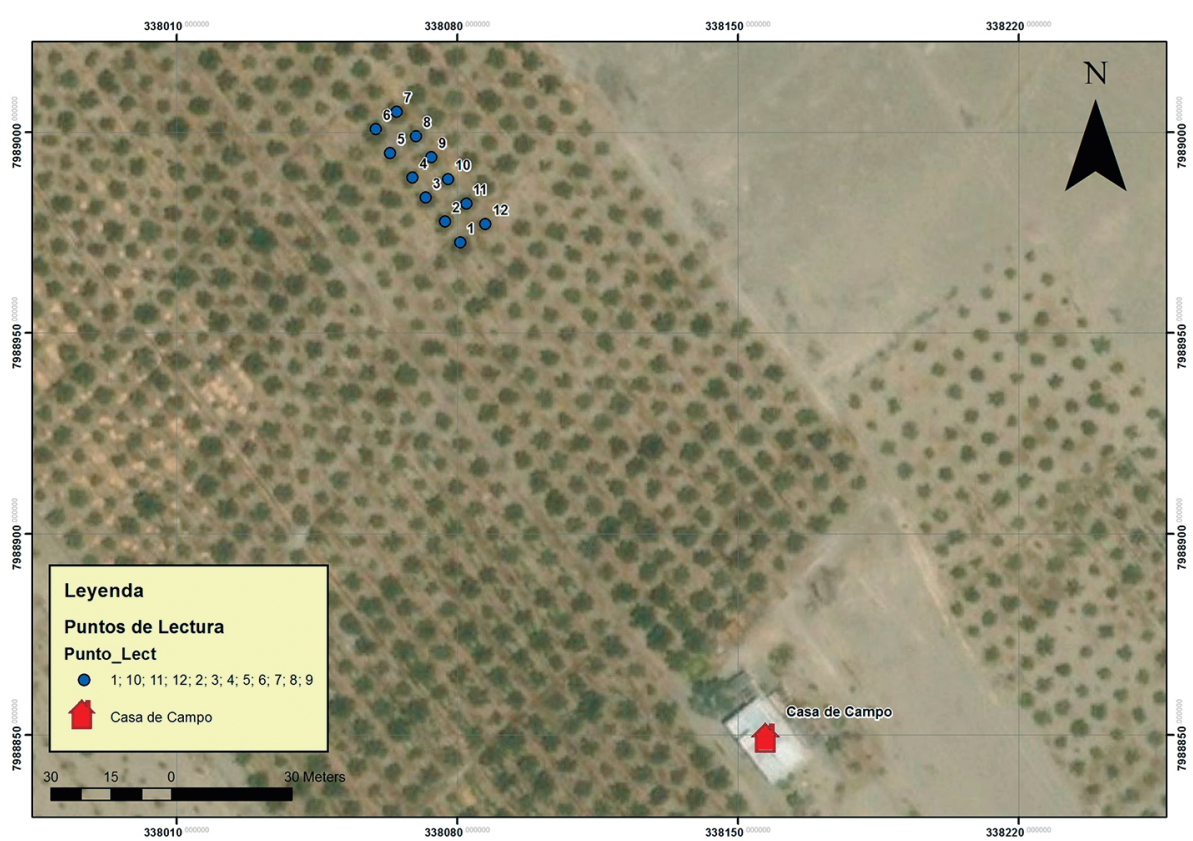

Figura 2. Ubicación del lugar donde se realizaron las mediciones en campo.

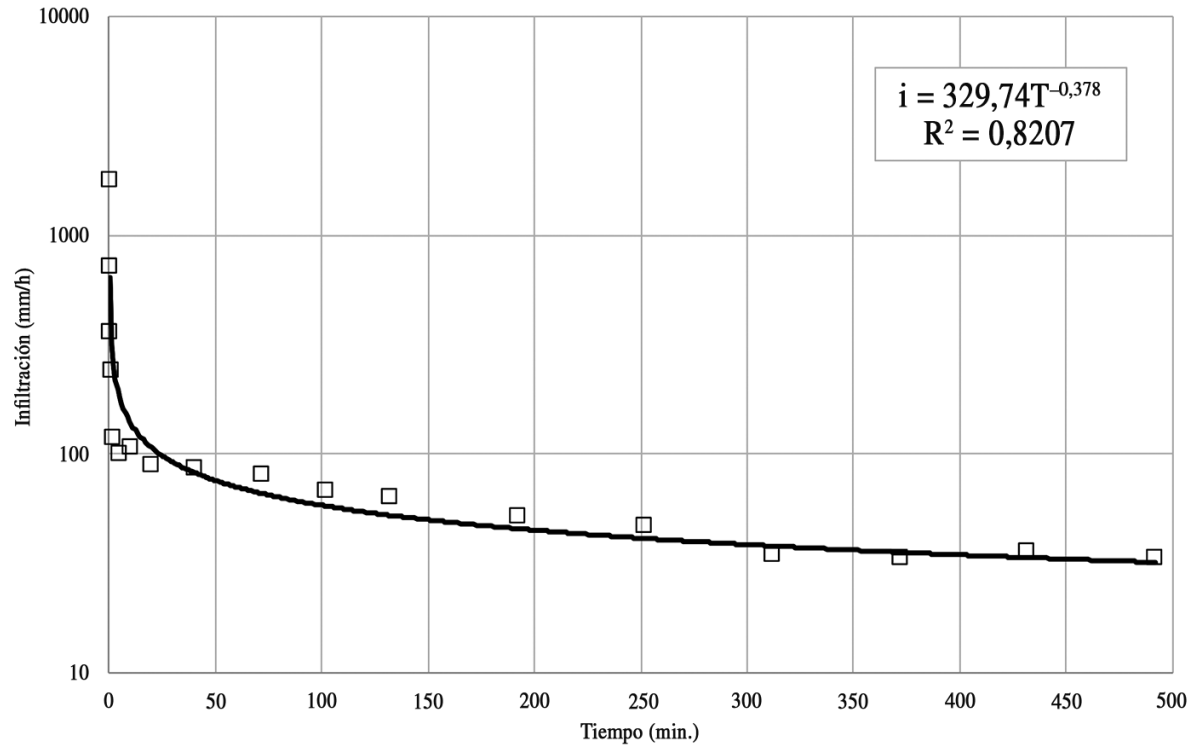

Figura 3. Curva de infiltración.

Pese a ello, existen varias formas de riego, pero no hay una planificación adecuada de los sistemas de riego, en especial en el distrito de La Yarada, debido a que crece la demanda de áreas agrícolas, requiriendo una mayor cantidad de agua.

Los estadios de crecimiento del olivo son: (1) Inducción floral, en la cual se identifica el momento en que se transforma la yema vegetativa en floral. (2) Diferenciación floral: comienza en el centro de la yema. Primero se desarrollan los pétalos, luego los sépalos, los estambres y el pistilo. Este proceso completo tarda de 4 a 5 semanas. (3) Aparición de racimos florales, con inflorescencias en las coberturas formadas por 
las hojas viejas. (4) Hinchazón del botón floral, redondeándose e inclinándose mediante un péndulo corto. (5) Floración: la inflorescencia comienza a crecer rápidamente, y el tamaño final lo alcanzan antes de septiembre u octubre. (6) Fructificación: aparecen los primeros frutos. Es la fecundación de la flor que mediante el proceso de polinización se convierte en fruto, el ovario fecundado se agranda y se hace notorio. (7) Maduración: los frutos adquieren el color típico de su variedad.

Para las mediciones en campo se utilizó un medidor de humedad TDR 350, basado en tecnología de medición en el dominio del tiempo, y un porómetro de hoja, para medir la conductancia estomática (g) de las hojas usando la técnica del estado estacionario (esta técnica mide la presión de vapor y el flujo de vapor sobre la superficie de la hoja). Este equipo permite obtener de forma automática lecturas de la conductancia estomática, eliminando la subjetividad de las medidas al elegir el final de medida. Las lecturas de la conductividad estomático se obtienen en 30 segundos.

La conductancia estomática se midió en dos etapas: la primera en una planta representativa entre las 6:00 y las 18:00 horas, cada hora, con tres repeticiones por medición, realizando las medidas en hojas externas expuestas a la radiación y otra en la parte interior; y la segunda en forma intensiva en un bloque de 12 plantas seleccionadas en la zona central de la plantación, donde se hicieron mediciones consecutivas entre las 11:00 y 14:00 horas del día. En forma paralela, se midió el contenido de humedad en el suelo a una profundidad de $20 \mathrm{~cm}$.

\section{Resultados y discusión}

En la Figura 4 se muestran los resultados del contenido de humedad del suelo a una profundidad de $20 \mathrm{~cm}$, durante 78 horas de registro. Los días 14,27 y 62 se registraron riegos, lo cual se nota claramente en la curva de variación de contenido de humedad. El olivo en general se caracteriza por su bajo consumo de agua, y esto se debe principalmente a la baja conductancia hidráulica del xilema que permite al árbol evitar la pérdida de agua en días de alta demanda atmosférica (Fernández et al., 1997).

La temperatura de la planta es un indicador de la conductancia estomática, que refleja las tensiones de la humedad del suelo, con lo que se pueden optimizar los programas de riego (Yu et al., 2015). En la Figura 5 se muestra la variación de la temperatura registrada en el follaje al interior $\mathrm{Ti}$ $\left({ }^{\circ} \mathrm{C}\right)$ y al exterior en la parte superficial del follaje del olivo Te $\left({ }^{\circ} \mathrm{C}\right)$, durante 78 horas de registro. Asimismo, en la Figura 6 observamos la variación de la conductancia estomática en el follaje al interior gi $\left(\mathrm{mmoles} / \mathrm{m}^{2} \mathrm{~s}\right)$, al exterior en la parte

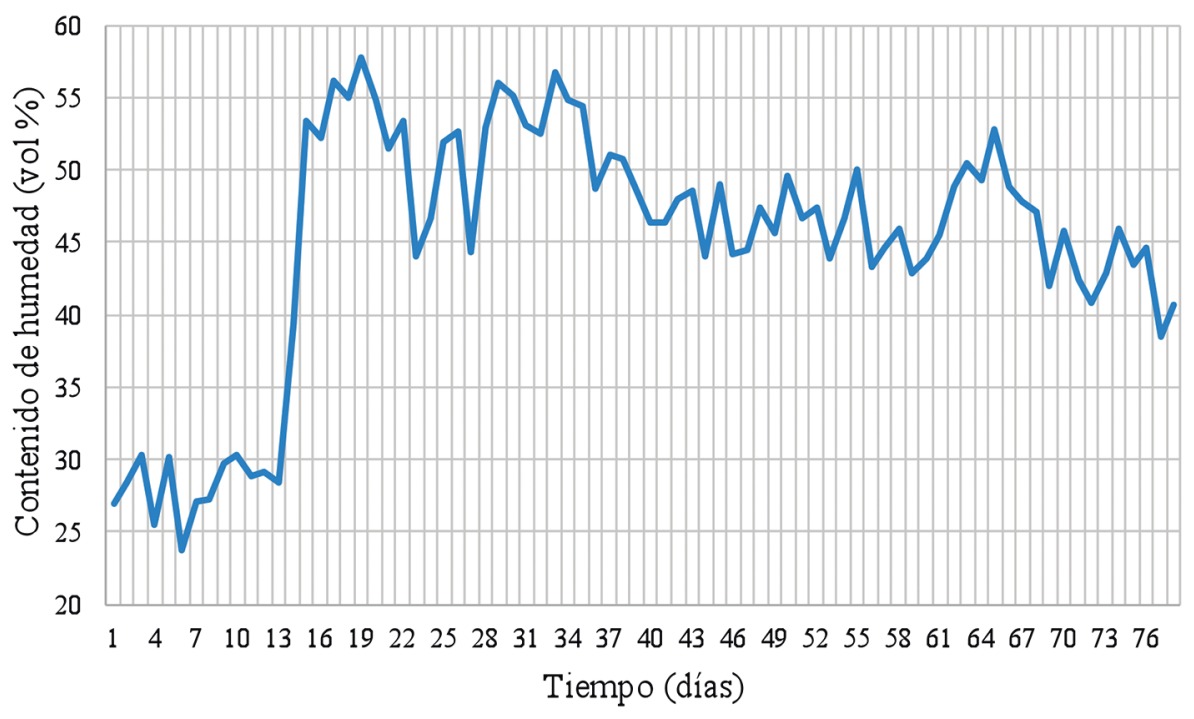

Figura 4. Variación del contenido de humedad registrada con TDR 350 a $20 \mathrm{~cm}$ de profundidad, durante 78 horas de registro, riegos reportados: horas 14, 27 y 62. 


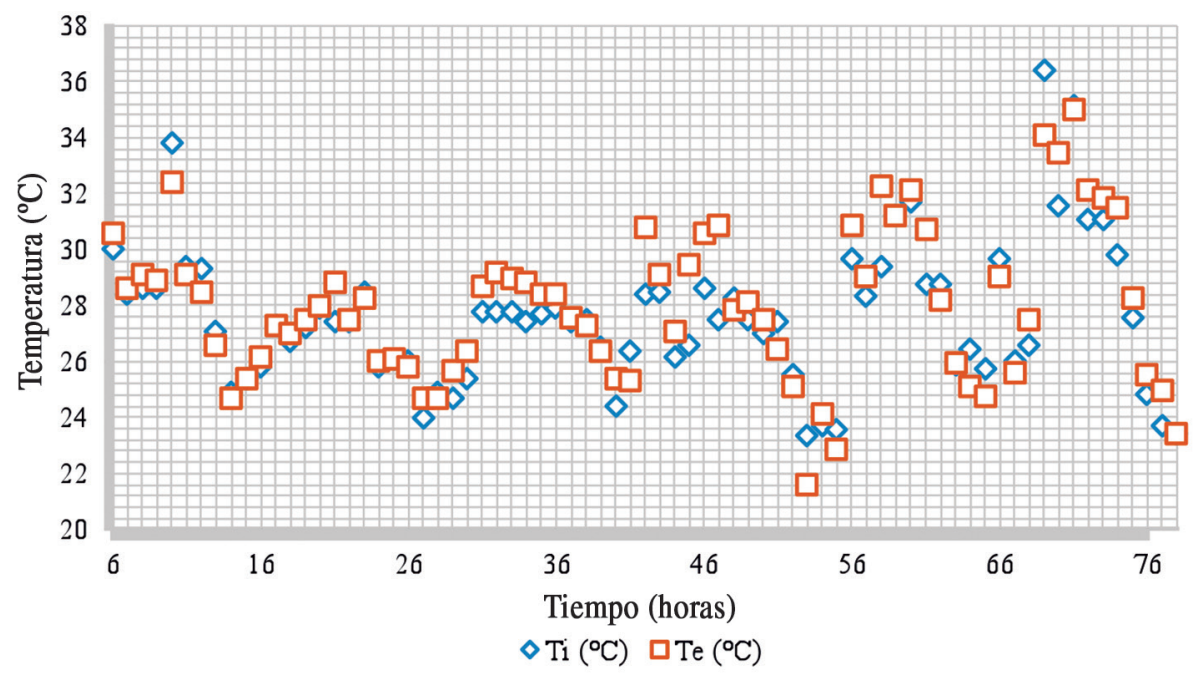

Figura 5. Variación de la temperatura registrada en el follaje al interior $\mathrm{Ti}\left({ }^{\circ} \mathrm{C}\right)$ y al exterior en la parte superficial del follaje del olivo $\mathrm{Te}\left({ }^{\circ} \mathrm{C}\right)$, durante 78 horas de registro.

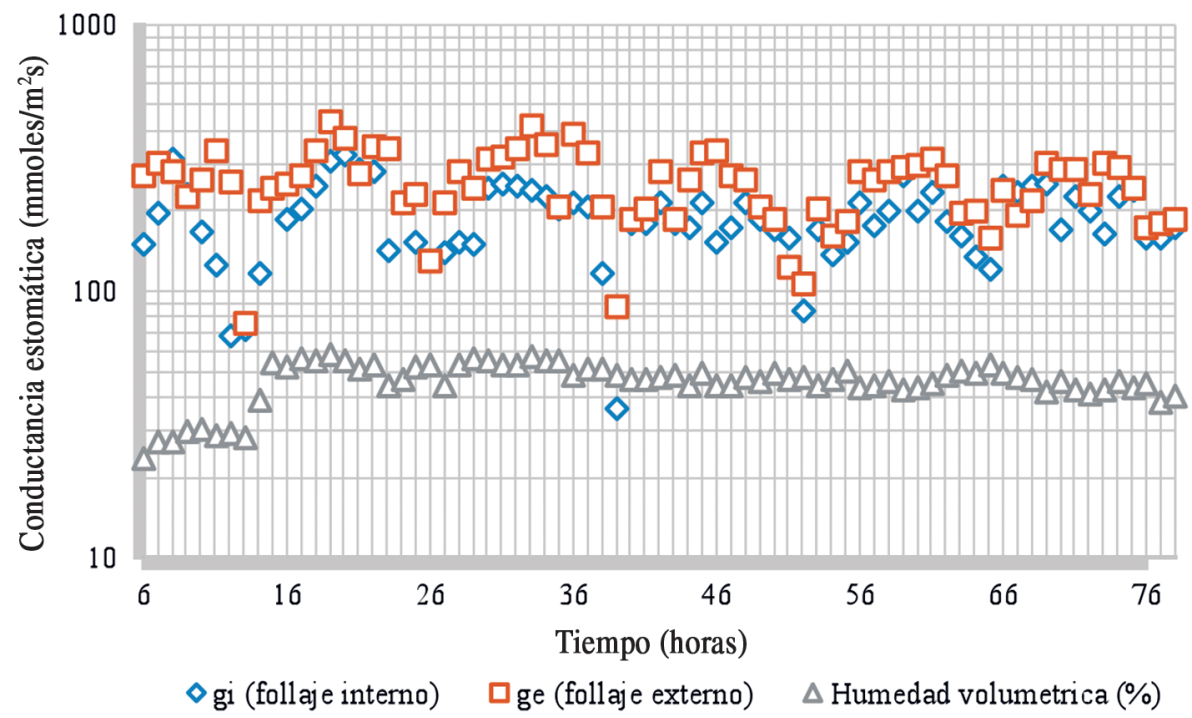

Figura 6. Variación de la conductancia estomática $\left(\mathrm{mmoles} / \mathrm{m}^{2} \mathrm{~s}\right)$ en el follaje al interior gi, al exterior en la parte superficial del follaje del olivo ge y variación del contenido de humedad en el suelo (vol\%), durante 78 horas de registro.

superficial del follaje del olivo $\mathrm{g}_{\mathrm{e}}\left(\mathrm{mmoles} \mathrm{m}^{2} \mathrm{~s}\right)$ y variación del contenido de humedad en el suelo (vol \%), durante 78 horas de registro.

En la Figura 7 se muestra la variación de la conductancia estomática en el follaje al interior gi $\left(\mathrm{mmoles} / \mathrm{m}^{2} \mathrm{~s}\right)$, al exterior en la parte superficial del follaje del olivo ge $\left(\mathrm{mmoles} / \mathrm{m}^{2} \mathrm{~s}\right)$, durante 12 horas de registro en forma diaria de 6 a 18 horas, durante 6 días.
La Figura 8 presenta la variación de la conductancia estomática en el follaje al interior gi $\left(\mathrm{mmoles} / \mathrm{m}^{2} \mathrm{~s}\right)$ y al exterior en la parte superficial del follaje del olivo ge (mmoles $\left./ \mathrm{m}^{2} \mathrm{~s}\right)$, en 12 horas de registro diario de 6 a 18 horas, promedio de 6 días del 31 marzo al 5 de abril. Las curvas polinomiales de ajuste utilizadas logran un ajuste importante para gi $=-2,4161 \mathrm{t}^{2}+53,013 \mathrm{t}-62,32 ;$ con $\mathrm{r}^{2}=0,8536 \mathrm{y}$

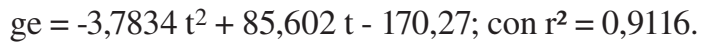



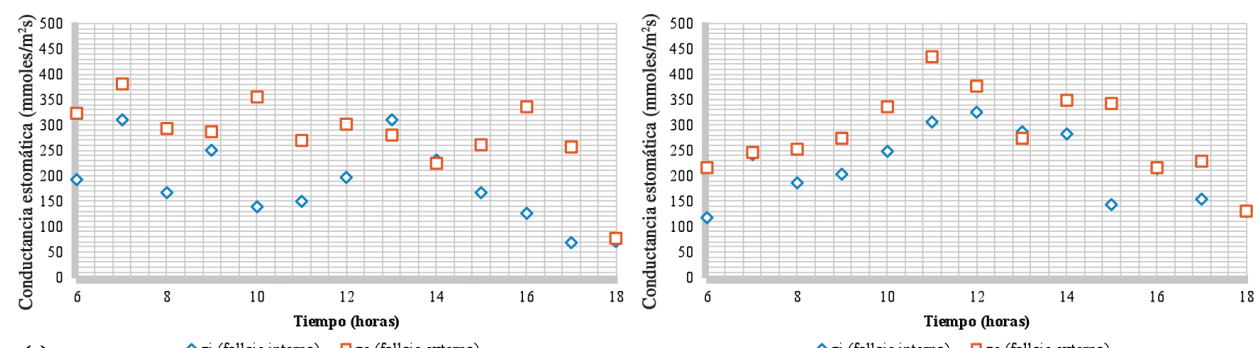

(a)

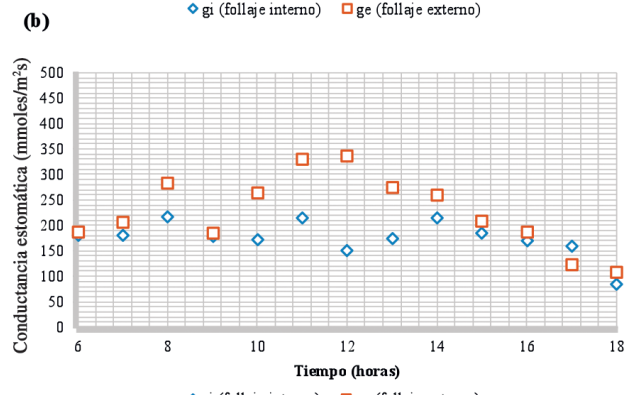

(c)

(d)

$\diamond$ gi (follaje interno) $\square$ ge (follaje externo)
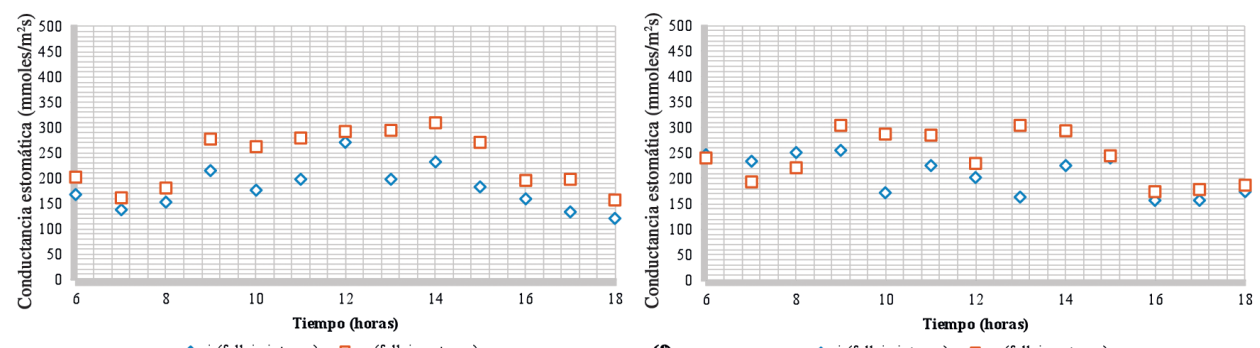

(e)

$\diamond$ gi (follaje interno) $\square$ ge (follaje externo)

(f)

$\diamond$ gi (follaje interno) पge (follaje externo)

Figura 7. Variación de la conductancia estomática $\left(\mathrm{mmoles} / \mathrm{m}^{2} \mathrm{~s}\right)$ en el follaje al interior gi, al exterior en la parte superficial del follaje del olivo ge, en 12 horas de registro diario de 6 a 18 horas, durante 6 días del 31 marzo al 5 de abril (a, b, c, d, e, f).

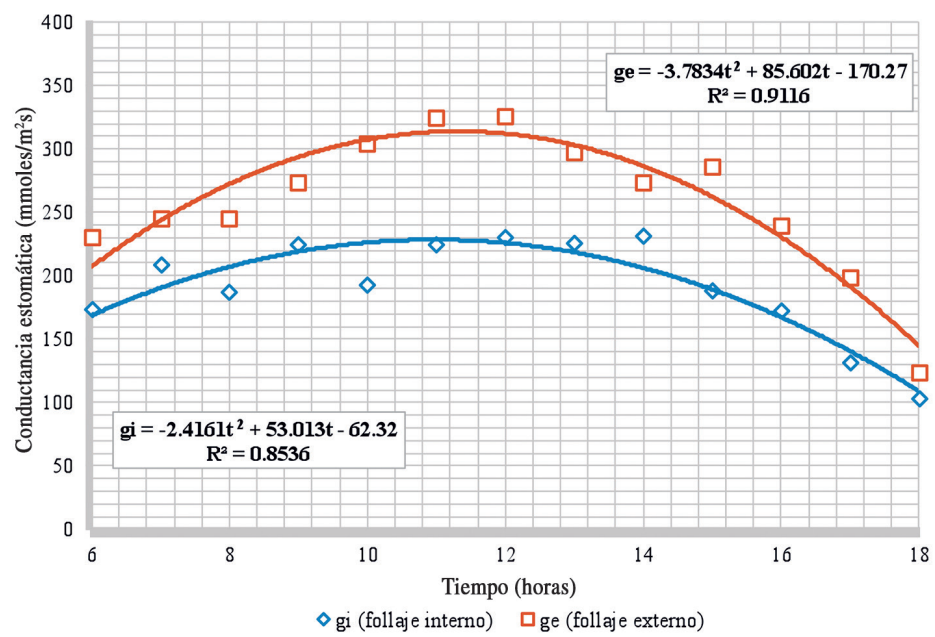

Figura 8. Variación de la conductancia estomática $\left(\mathrm{mmoles} / \mathrm{m}^{2} \mathrm{~s}\right)$ en el follaje al interior gi, al exterior en la parte superficial del follaje del olivo ge, en 12 horas de registro diario de 6 a 18 horas, promedio de 6 días del 31 marzo al 5 de abril. 
En el mes de junio, día 19, se realizó trabajo de campo para medir la conductancia en forma intensiva. Metodológicamente se tomaron 12 plantas en doble hilera, dispuestas 6 por 2, haciendo las mediciones entre las 11:00 y 14:00 horas para buscar los máximos valores de conductancia. En la Figura 9 se observa una tendencia típica de picos hacia las horas medias del día, encontrándose un valor máximo de $411 \mathrm{mmoles} / \mathrm{m}^{2} \mathrm{~s}$.

En cuanto a la temperatura y la conductancia, se logró un ajuste significativo gs $=20,744 \mathrm{~T}-349,99, \mathrm{r}^{2}$
$=0,6257$, lo que demuestra una relación entre estos parámetros. La tendencia es a mayor temperatura foliar mayor conductancia estomática, tal como se muestra en la Figura 10.

Según Yu et al. (2015), la temperatura de las hojas, la conductancia estomática y los índices de estrés difieren significativamente. La conductancia estomática disminuye con la temperatura media del dosel en los tratamientos de estrés por sequía tanto constante como en gradiente. En la Figura 11 se muestra la relación entre la conductancia estomática

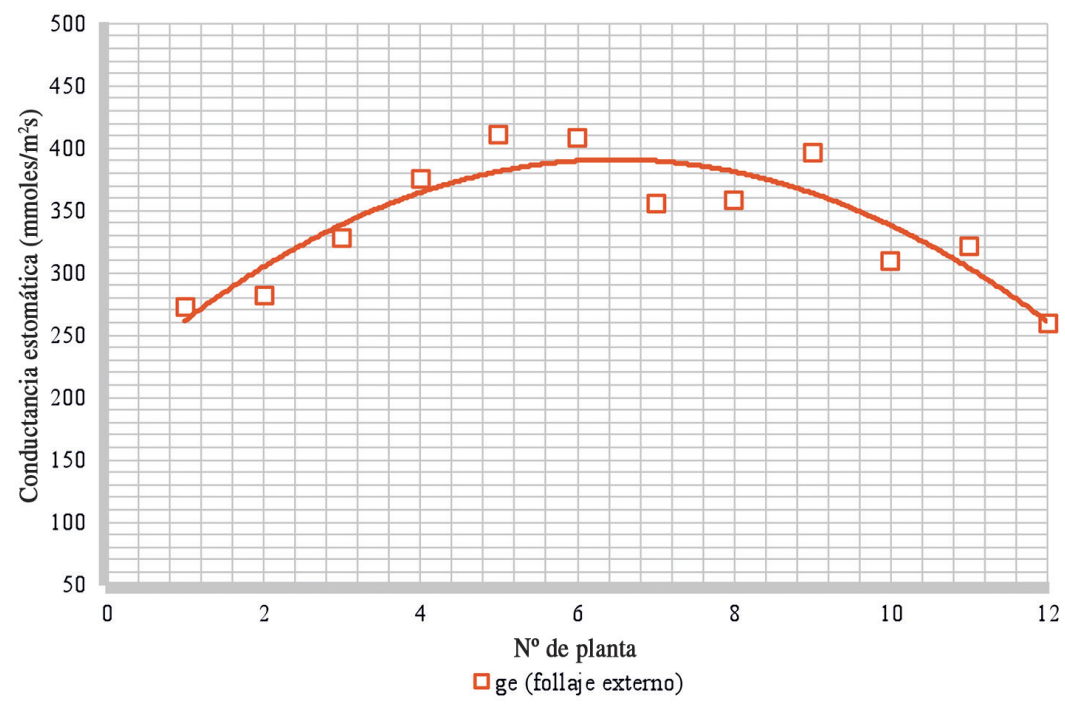

Figura 9. Variación de la conductancia estomática $\left(\mathrm{mmoles} / \mathrm{m}^{2} \mathrm{~s}\right)$ en el follaje al exterior en la parte superficial del follaje del olivo ge, en 3 horas de registro diario de 11 a 14 horas.

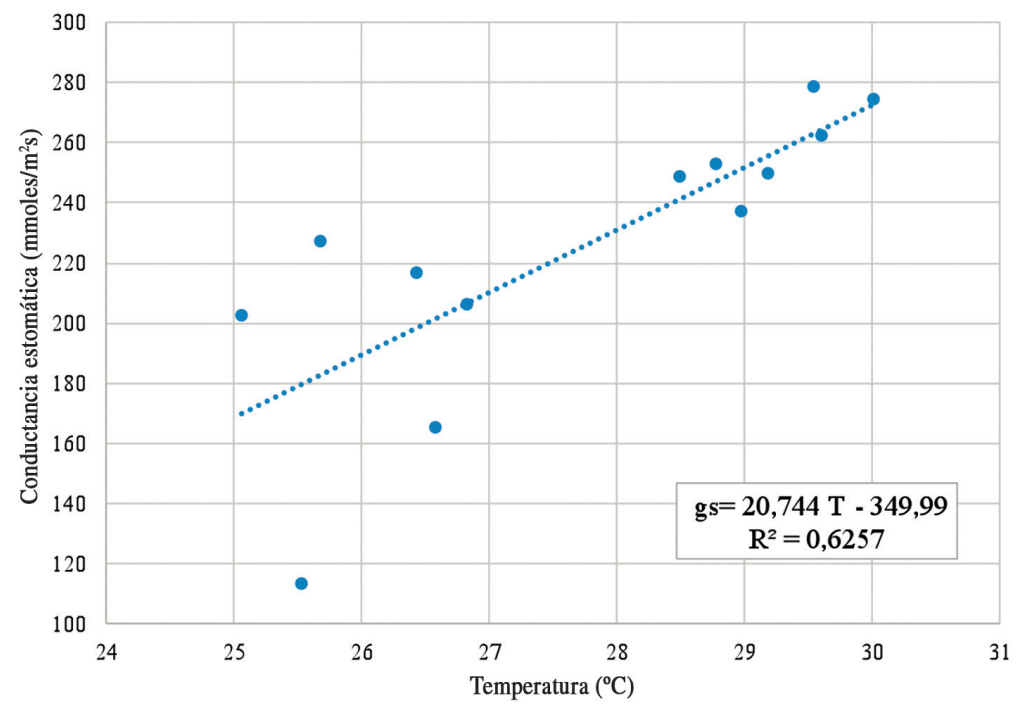

Figura 10. Variación de la conductancia estomática en función de la temperatura foliar. 
tomando en cuenta los valores del follaje al interior (gi) y exterior (ge) en mmoles $/ \mathrm{m}^{2} \mathrm{~s}$, y el contenido de humedad volumétrico en ambos casos valores promedio horario entre las 6 y 18 horas, cada hora, por un período de 6 días. El resultado arroja que no existe correlación entre estos elementos, lo que se explica en la variación del contenido de humedad que en ese período no es significativo (entre las 6 y 18 horas de un día), mientras que la conductancia estomática sí varía en dicho lapso, influenciada preferentemente por la temperatura de las hojas del olivo.

\section{Conclusión}

El olivo se caracteriza por su bajo consumo de agua, debido a la baja conductancia hidráulica del xilema que le permite evitar la pérdida de agua en días de alta demanda atmosférica. La temperatura de la superficie foliar de la planta es un indicador de la conductancia estomática. Según las mediciones y correlaciones buscadas, la temperatura y la conductancia arrojaron un ajuste significativo, y la tendencia es a mayor temperatura foliar mayor conductancia estomática. Asimismo, se encontró que no existe correlación entre la conductancia estomática tomando en cuenta los valores del follaje al interior (gi) y exterior (ge) en mmoles $/ \mathrm{m}^{2} \mathrm{~s}$, y el contenido de humedad volumétrico del suelo. Según mediciones intensivas de conductancia estomática, se observa una tendencia típica de picos hacia las horas medias del día, y se encuentra un valor máximo de 411 $\mathrm{mmoles} / \mathrm{m}^{2} \mathrm{~s}$, para el período de maduración de frutos en el olivo.

\section{Agradecimiento}

Este trabajo ha sido desarrollado en el marco del proyecto de investigación "Uso de Vehículos Aéreos no Tripulados para obtener la humedad del suelo en el olivo con fines de programación de riego en tiempo real en La Yarada, Tacna, Perú”, financiado con fondos de canon, sobrecanon y regalías mineras de la Universidad Nacional Jorge Basadre Grohmann, Tacna, Perú.

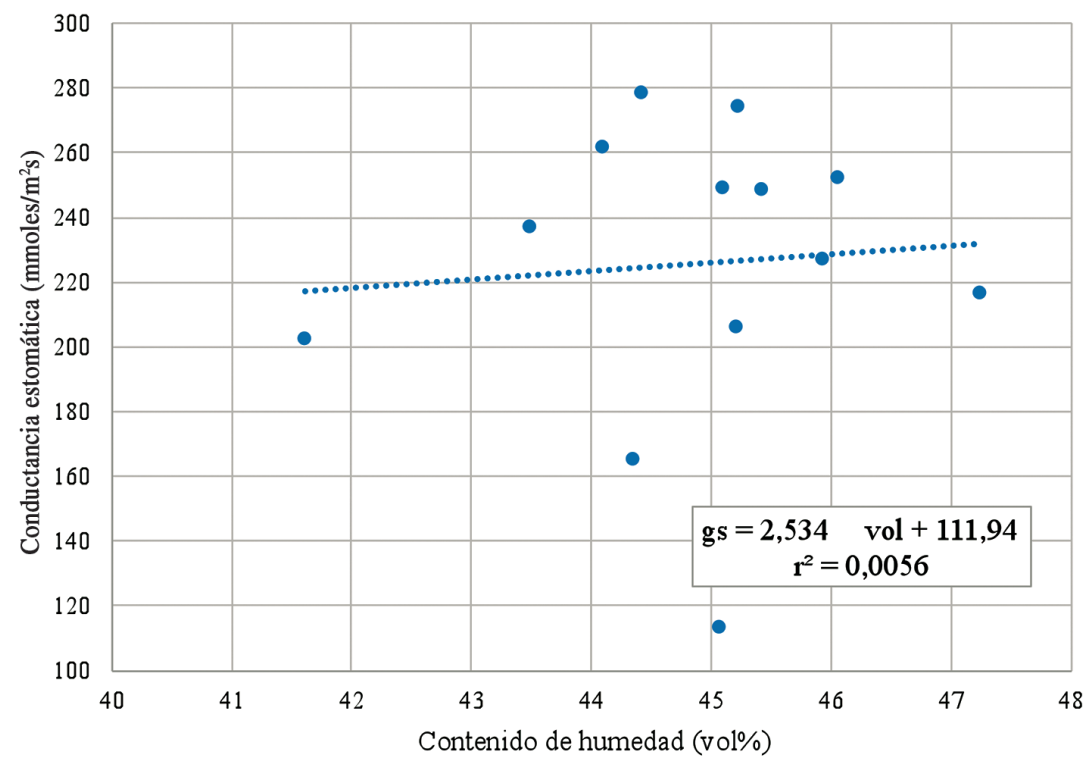

Figura 11. Relación entre la conductancia estomática promedio tomando en cuenta los valores del follaje al interior (gi) y exterior (ge) en mmoles $/ \mathrm{m}^{2} \mathrm{~s}$, y el contenido de humedad volumétrico. 


\section{Literatura Citada}

Ashraf, M.; Harris, P.J.C.

2013. Photosynthesis under stressful environments: An overview. Photosynthetica, 51 (2): 163-190.

Bacelar, E.A.; Correia, C.M.; Moutinho-Pereira, J.M.; Goncalves,

B.C.; Lopes, J.I.; Torres-Pereira, J.M.G.

2004. Sclerophylly and leaf anatomical traits of five fieldgrown olive cultivars growing under drought conditions. Tree Physiology, 24 (2): 233-239.

Ball, J.T.; Woodrow, I.E.; Berry, J.A.

1987. A Model Predicting Stomatal Conductance and its Contribution to the Control of Photosynthesis under Different Environmental Conditions. En J. Biggins (Ed.). Progress in Photosynthesis Research. Vol. IV. Martin us Nijhoff Publishers. Dordrecht, Netherlands. pp. 221-224.

Beerling, D.J.

2015. Gas valves, forests and global change: a commentary on Jarvis (1976) "The interpretation of the variations in leaf water potential and stomatal conductance found in canopies in the field". Philosophical Transactions of the Royal Society B: Biological Sciences, 370 (1666): 20140311-20140311.

Buckley, T.N.

2017. Modeling Stomatal Conductance. Plant Physiology, 174 (2): 572-582.

Dell' Amico-Rodriguez, J.; Morales-Guevara, D.M.

2017. Comportamiento de la conductancia estomática de dos variedades de tomate cubanas en condiciones de campo y riego limitado. Cultivos Tropicales, 38: 137-144.

Fernández, J.E.; Moreno, F.; Girón, I.F.; Blázquez, O.M.

1997. Stomatal control of water use in olive tree leaves. Plant and Soil, 190 (2): 179-192.

Giorio, P.; Sorrentino, G.; D’Andria, R.

1999. Stomatal behaviour, leaf water status and photosynthetic response in field-grown olive trees under water deficit. Environmental and Experimental Botany, 42 (2): 95-104.

Lauer, J.G.; Bijl, C.G.; Grusak, M.A.; Baenziger, P.S.; Boote,

K.; Lingle, S.; Willcox, M.C.

2012. The Scientific Grand Challenges of the 21st Century for the Crop Science Society of America. Crop Science, 52 (3): 1003.

Li, C.; Ng, C.K.-Y.; Fan, L.-M.

2015. MYB transcription factors, active players in abiotic stress signaling. Environmental and Experimental Botany, 114: 80-91.
Manzoni, S.; Vico, G.; Palmroth, S.; Porporato, A.; Katul, G. 2013. Optimization of stomatal conductance for maximum carbon gain under dynamic soil moisture. Advances in Water Resources, 62: 90-105.

Mencuccini, M.

2014. Temporal scales for the coordination of tree carbon and water economies during droughts. Tree Physiology, 34 (5): 439-442.

Moriana, A.; Villalobos, F.J.; Fereres, E.

2002. Stomatal and photosynthetic responses of olive (Olea europaea L.) leaves to water deficits. Plant, Cell and Environment, 25 (3): 395-405.

Nielsen, D.C.; Nelson, N.O.

1998. Black Bean Sensitivity to Water Stress at Various Growth Stages. Crop Science, 38 (2): 422.

Orgaz, F.; Villalobos, F.J.; Testi, L.; Fereres, E.

2007. A model of daily mean canopy conductance for calculating transpiration of olive canopies. Functional Plant Biology, 34 (3): 178.

Pino, E.; Tacora, P.; Steenken, A.; Alfaro, L.; Valle, A.; Chávarri, E.; Mejía, J.

2017. Efecto de las características ambientales y geológicas sobre la calidad del agua en la cuenca del río Caplina, Tacna, Perú. Tecnología y Ciencias del Agua, 08 (06): 77-99.

Prasch, C.M.; Sonnewald, U.

2015. Signaling events in plants: Stress factors in combination change the picture. Environmental and Experimental Botany, 114, 4-14.

Tognetti, R.; Giovannelli, A.; Lavini, A.; Morelli, G.; Fragnito, F.; D'Andria, R.

2009. Assessing environmental controls over conductances through the soil-plant-atmosphere continuum in an experimental olive tree plantation of southern Italy. Agricultural and Forest Meteorology, 149 (8): 1229-1243.

Trenberth, K.E., Dai, A.; van der Schrier, G.; Jones, P.D.; Barichivich, J.; Briffa, K.R.; Sheffield, J.

2014. Global warming and changes in drought. Nature Climate Change, 4 (1): 17-22.

Wang, F.; Kang, S.; Du, T.; Li, F.; Qiu, R.

2011. Determination of comprehensive quality index for tomato and its response to different irrigation treatments. Agricultural Water Management, 98 (8): 1228-1238.

Yu, M.-H.; Ding, G.-D.; Gao, G.-L.; Zhao, Y.-Y.; Yan, L.; Sai, K. 2015. Using Plant Temperature to Evaluate the Response of Stomatal Conductance to Soil Moisture Deficit. Forests, 6 (10): 3748-3862. 